DOI: 10.12731/2658-6649-2020-12-5-11-15

УДК 51-7

\title{
ПРОГНОЗИРОВАНИЕ ЛИПОФИЛЬНЫХ СВОЙСТВ ПРОИЗВОДНЫХ АДАМАНТАНА
}

\section{Осипов А.Л., Трушина В.П.}

B статье исследуются QSPR модели предсказания липофильности химических веществ семейства адамантанов. Исследование параметра липофильности осуществляется с помощью разработанных нелинейных моделей с использованием абсолютной энтропии. Проведены вычислительные эксперименты, показывающие высокую эффективность предложенных QSPR зависимостей.

Ключевые слова: предсказание; липофильность; абсолютная энтропия; регрессионный анализ; QSPR.

\section{PREDICTION OF LIPOPHILIC PROPERTIES OF ADAMANTANE DERIVATIVES}

\author{
Osipov A.L., Trushina V.P.
}

The article explores QSPR models for predicting the lypophilicity of chemicals in the adamantane family. The study of the lypophilicity parameter is carried out using the developed nonlinear models using absolute entropy. Computational experiments have been performed, showing the high efficiency of the proposed QSPR dependencies.

Ключевые слова: prediction; lipophilicity; absolute entropy; regression analysis; $Q S P R$.

\section{Введение}

Гидрофобные взаимодействия (липофильность) действуют в водной среде и имеют энтропийную природу. Поэтому изучение липофильности через абсолютную энтропию является весьма перспективным направлением для производных адамантана, которые обладают ярко выраженной биологической активностью. Актуальность статьи заключается в конструировании новых QSPR моделей для предсказания параметра ли- 
пофильности с учетом только одного фактора, а именно, абсолютной энтропии $(S)$.

\section{Методы исследования}

Методы исследований включают: хемоинформатику, корреляционную зависимость между структурой и лекарственными свойствами (QSPR); программирование; моделирование и прогнозирование.

\section{Результаты исследования}

В качестве экспериментальной выборки были взяты 24 химических вещества из семейства адамантанов [1, с. 133], представленные в таблице 1 своими физико-химическими параметрами.

Таблицуа 1.

Выборка производных адамантана

\begin{tabular}{|c|c|c|}
\hline № & $\log P$ & $S$ \\
\hline 1 & 0,52 & 1474,3408 \\
\hline 2 & 0,47 & 1474,3408 \\
\hline 3 & 2,7 & 1769,8676 \\
\hline 4 & 3,19 & 1906,2354 \\
\hline 5 & 3,19 & 1906,2354 \\
\hline 6 & 2,86 & 2253,4610 \\
\hline 7 & 2,99 & 2203,7482 \\
\hline 8 & 3,26 & 1806,2676 \\
\hline 9 & 3,26 & 1806,2676 \\
\hline 10 & 3,53 & 1816,0776 \\
\hline 11 & 2,44 & 300,8960 \\
\hline 12 & 1,69 & 333,3600 \\
\hline 13 & 2,03 & 365,8340 \\
\hline 14 & 1,55 & 606,2600 \\
\hline 15 & 1,04 & 479,6580 \\
\hline 16 & 1,45 & 437,9920 \\
\hline 17 & 1,83 & 470,4680 \\
\hline 18 & 1,42 & 559,8670 \\
\hline 19 & 1,67 & 424,0180 \\
\hline 20 & 1,63 & 370,0910 \\
\hline 21 & 1,92 & 402,5660 \\
\hline 22 & 0,92 & 462,3600 \\
\hline 23 & 1,21 & 494,8340 \\
\hline 24 & 1,55 & 422,7340 \\
\hline & & \\
\hline
\end{tabular}


B работе [1, с. 134] представлена QSPR модель предсказания параметра липофильности на основе фактора, связанного с абсолютной энтропией $\log P=10,29-0,038 S+0,00004 S^{2}$. Параметры модели следующие: объем выборки $n=16$; коэффициент детерминации $R^{2}=0,96$; стандартная ошибка $S D=0,37$; критерий Фишера $F=146,72$. В этой работе выявлено, что коэффициент в модели при $S^{2}$ значим и находится в пределах $[0,000033 ; 0,000048]$, причем сам коэффициент равен 0,0004 и не попадает в этот интервал. Скорее всего он равен 0,00004 и модель имеет вид $\log P=$ $10,29-0,038 S+0,00004 S^{2}$. Данная модель неэффективно предсказывает параметр липофильности для семейства адамантанов. Для первых десяти соединений она дает очень высокие показатели липофильности, например, для соединения с номером шесть, предсказанное значение липофильности оказалось равным 127,7819 (реальное значение равно 2,86). Для других девяти соединений предсказанные значения находились в интервале от 41 до 120 , хотя истинные значения липофильности были меньше 3,6.

B статье разработаны следующие QSPR значимые модели предсказания параметра липофильности, представленные в таблице 2.

Таблицуа 2.

Характеристики моделей для предсказания липофильности

\begin{tabular}{|l|c|c|c|c|}
\hline \multicolumn{1}{|c|}{ Модель } & $R^{2}$ & $F$ & $S D$ & $n$ \\
\hline $\begin{array}{l}\log P=7,2131-0,02292 S+0,000022 S^{2} \\
\text { для эле- }\end{array}$ & 0,566 & 7,179 & 0,284 & 14 \\
\hline $\begin{array}{l}\log P=-40,1696+0,04375 S+0,000011 S^{2} \text { для эле- } \\
\text { ментов выборки со значением энтропии } S<1000\end{array}$ & 0,969 & 107,77 & 0,228 & 10 \\
\hline $\log P=0,15897 * S^{0,3871}$ & 0,634 & 32,91 & 0,459 & 21 \\
\hline
\end{tabular}

Первые две модели можно записать в следующем виде

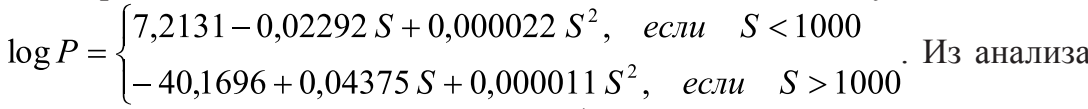
моделей видно, что стандартная ошибка у двух приведенных выше моделей лучше, чем в работе [1, с. 134]. Для соединения с номером шесть прогнозное значение по моделям оказалось равным 2,56 и 3,156. Расчет по моделям проводился с помощью MS Excel и статистического пакета SPSS [2, c. 57].

\section{Обсуждение}

Предлагаемая методология на основе QSPR позволяет эффективно предсказывать липофильность для семейства адамантанов по достаточно 
простым моделям. Представленные QSPR модели можно рекомендовать для практического использования.

\section{Заключение}

Предложены модели для исследования взаимосвязи параметра липофильности семейства адамантанов через их абсолютную энтропию. Проведено сравнение полученных моделей с уже существующими подходами.

Информация о конфликте интересов. Отсутствие конфликта интересов.

Информация о спонсорстве. Исследование не имело спонсорской поддержки.

\section{Сиисок литературы}

1. Коноваленко С.П., Исаев П.П. Прогнозирование гидрофобных свойств биофармацевтических препаратов // Известия ЮФУ. Технические науки. №9 (110). 2010. C. 131-135.

2. Бюль А., Цефель П. SPSS: искусство обработки информации. СПб: ДиаСофтЮП. 2005. 608 c.

\section{References}

1. Konovalenko S.P., Isaev P.P. Izvestiya YuFU. Tekhnicheskie nauki. №9 (110). 2010, pp. 131-135.

2. Bul A., Zefel P. SPSS: iskusstvo obrabotki informatsii [SPSS: the art of information processing]. SPb: DiaSoftYuP. 2005. 608 p.

\section{ДАННЫЕ ОБ АВТОРАХ}

Осипов Александр Леонидович, доцент, кандидат технических наук ФГБОУ ВО Новосибирский государственный университет экономики и управления

ул. Каменская, 56, г. Новосибирск, 630099, Российская Федерация alosip@mail.ru

Трушина Вероника Павловна, старший преподаватель ФГБОУ ВО Новосибирский государственный университет экономики и управления

ул. Каменская, 56, г. Новосибирск, 630099, Российская Федераџия veronika07-92@mail.ru 
DATA ABOUT THE AUTHORS

Osipov Alexander Leonidovich, Associate Professor, Candidate of Technical Sciences

Novosibirsk State University of Economics and Management 56, Kamenskaya str., Novosibirsk, 630099, Russian Federation alosip@mail.ru

Trushina Veronica Pavlovna, senior teacher

Novosibirsk State University of Economics and Management 56, Kamenskaya str., Novosibirsk, 630099, Russian Federation veronika07-92@mail.ru 